\title{
The Shape of Multidimensional Gravity: Non-relativistic Limit
}

\author{
Maxim Eingorn and Alexander Zhuk \\ Astronomical Observatory and Department of Theoretical Physics, Odessa National University, \\ Street Dvoryanskaya 2, Odessa 65082, Ukraine
}

\begin{abstract}
It is found the exact solution of the Poisson equation for the multidimensional space with topology $M_{3+d}=\mathbb{R}^{3} \times T^{d}$. This solution describes smooth transition from the Newtonian behavior $1 / r_{3}$ for distances bigger than periods of tori (the extra dimension sizes) to multidimensional behavior $1 / r_{3+d}^{1+d}$ in opposite limit. In the case of one extra dimension $d=1$, the gravitational potential is expressed via compact and elegant formula. Obtained formula is applied to an infinitesimally thin shell, a shell, a sphere and two spheres to show deviations from the Newtonian expressions. It is shown that the corrections to the gravitational constant in the Cavendish-type experiment can be within the measurement accuracy of Newton's gravitational constant $G_{N}$. It is proposed models where the test masses are smeared over some (or all) extra dimensions. In 10-dimensional spacetime with 3 smeared extra dimensions, it is shown that the size of 3 rest extra dimensions can be enlarged up to submillimeter for the case of $1 \mathrm{TeV}$ fundamental Planck scale $M_{P l(10)}$. In the models where all extra dimensions are smeared, the gravitational potential exactly coincides with the Newtonian one. Nevertheless, the hierarchy problem can be solved in these models.
\end{abstract}

Keywords: Multidimensional gravity, Newton's law, hierarchy problem

PACS: $04.50 .-\mathrm{h}, 11.25 . \mathrm{Mj}, 98.80 .-\mathrm{k}$

\section{INTRODUCTION}

There are two well-known problems which are related to each other. They are the discrepancies in gravitational constant experimental data and the hierarchy problem. Discrepancies (see e.g. Figure 2 in the "CODATA Recommended Values of the Fundamental Constants: 2006") are usually explained by extreme weakness of gravity. It is very difficult to measure the Newton's gravitational constant $G_{N}$. Certainly, for this reason geometry of an experimental setup can effect on data. However, it may well be that, the discrepancies can also be explained (at least partly) by underlying fundamental theory. Formulas for an effective gravitational constant following from such theory can be sensitive to the geometry of experiments. For example, if correction to the Newton's gravitational potential has the form of Yukawa potential, then the force due to this potential at a given minimum separation per unit test-body mass is least for two spheres and greatest for two planes (see e.g.[1]). The hierarchy problem - the huge gap between the electroweak scale $M_{E W} \sim 10^{3} \mathrm{GeV}$ and the Planck scale $M_{P l(4)}=1.2 \times 10^{19} \mathrm{GeV}$ can be also reformulated in the following manner: why is gravity so weak? The smallness of $G_{N}$ is the result of relation $G_{N}=M_{P l(4)}^{-2}$. The natural explanation was proposed in [2, 3]: the gravity is strong: $G_{\mathscr{D}}=M_{P l(\mathscr{D})}^{-(2+d)} \sim M_{E W}^{-(2+d)}$ and it happens in $(\mathscr{D}=4+d)$ dimensional spacetime. It becomes weak when gravity is "smeared" over large extra dimensions: $G_{N} \sim G_{\mathscr{D}} / V_{d}$ where $V_{d}$ is a volume of internal space. To shed light on both 
of these problems from new standpoint we intend to investigate multidimensional gravity in non-relativistic limit.

\section{MULTIDIMENSIONAL GRAVITATIONAL POTENTIALS}

It is of interest to generalize the well-known Newton's gravitational potential $\varphi\left(r_{3}\right)=$ $-G_{N} m / r_{3}\left(r_{3}=\left|\mathbf{r}_{3}\right|\right.$ is magnitude of a radius vector in three-dimensional space) to multidimensional case. Clearly, the result depends on topology of investigated models. We consider models where $(D=3+d)$-dimensional spatial part of factorizable geometry is defined on a product manifold $M_{D}=\mathbb{R}^{3} \times T^{d} . \mathbb{R}^{3}$ describes three-dimensional flat external (our) space and $T^{d}$ is a torus which corresponds to $d$-dimensional internal space with volume $V_{d}$. Let $b \sim V_{d}^{1 / d}$ be a characteristic size of extra dimensions. Then, Gauss's flux theorem leads to the following asymptotes for gravitational potential (see e.g. [3]): $\varphi \sim 1 / r_{3}$ for $r_{3}>>b$ and $\varphi \sim 1 / r_{3+d}^{1+d}$ for $r_{3+d}<<b$ where $r_{3+d}$ is magnitude of a radius vector in $(3+d)$-dimensional space.

To get the exact expression for $D$-dimensional gravitational potential, we start with the Poisson equation:

$$
\triangle_{D} \varphi_{D}=S_{D} G_{\mathscr{D}} \rho_{D}\left(\mathbf{r}_{D}\right),
$$

where $S_{D}=2 \pi^{D / 2} / \Gamma(D / 2)$ is a total solid angle (square of $(D-1)$-dimensional sphere of a unit radius), $G_{\mathscr{D}}$ is a gravitational constant in $(\mathscr{D}=D+1)$-dimensional spacetime and $\rho_{D}\left(\mathbf{r}_{D}\right)=m \delta\left(x_{1}\right) \delta\left(x_{2}\right) \ldots \delta\left(x_{D}\right)$. In the case of topology $\mathbb{R}^{D}$, Eq. (1) has the following solution:

$$
\varphi_{D}\left(\mathbf{r}_{D}\right)=-\frac{G_{\mathscr{D}} m}{(D-2) r_{D}^{D-2}}, \quad D \geq 3 .
$$

This is the unique solution of Eq. (1) which satisfies the boundary condition: $\lim _{r_{D} \rightarrow+\infty} \varphi_{D}\left(\mathbf{r}_{D}\right)=0$. Gravitational constant $G_{\mathscr{D}}$ in (1) is normalized in such a way that the strength of gravitational field (acceleration of a test body) takes the form: $-d \varphi_{D} / d r_{D}=-G_{\mathscr{D}} m / r_{D}^{D-1}$.

If topology of space is $\mathbb{R}^{3} \times T^{d}$, then it is natural to impose periodic boundary conditions in the directions of the extra dimensions: $\varphi_{D}\left(\mathbf{r}_{3}, \xi_{1}, \xi_{2}, \ldots, \xi_{i}, \ldots, \xi_{d}\right)=$ $\varphi_{D}\left(\mathbf{r}_{3}, \xi_{1}, \xi_{2}, \ldots, \xi_{i}+a_{i}, \ldots, \xi_{d}\right), \quad i=1, \ldots, d$, where $a_{i}$ denotes a period in the direction of the extra dimension $\xi_{i}$. Then, Poisson equation has solution (cf. also with [3, 4]):

$$
\begin{aligned}
& \varphi_{D}\left(\mathbf{r}_{3}, \xi_{1}, \ldots, \xi_{d}\right)=-\frac{G_{N} m}{r_{3}} \sum_{k_{1}=-\infty}^{+\infty} \ldots \sum_{k_{d}=-\infty}^{+\infty} \exp \left[-2 \pi\left(\sum_{i=1}^{d}\left(\frac{k_{i}}{a_{i}}\right)^{2}\right)^{1 / 2} r_{3}\right] \\
& \times \cos \left(\frac{2 \pi k_{1}}{a_{1}} \xi_{1}\right) \ldots \cos \left(\frac{2 \pi k_{d}}{a_{d}} \xi_{d}\right) .
\end{aligned}
$$

To get this result we, first, use the formula $\delta\left(\xi_{i}\right)=\frac{1}{a_{i}} \sum_{k=-\infty}^{+\infty} \cos \left(\frac{2 \pi k}{a_{i}} \xi_{i}\right)$ and, second, put the following relation between gravitational constants in four- and $\mathscr{D}$-dimensional 
spacetimes:

$$
\frac{S_{D}}{S_{3}} \cdot \frac{G_{\mathscr{D}}}{\prod_{i=1}^{d} a_{i}}=G_{N} .
$$

The letter relation provides correct limit when all $a_{i} \rightarrow 0$. In this limit zero modes $k_{i}=0$ give the main contribution and we obtain $\varphi_{D}\left(\mathbf{r}_{3}, \xi_{1}, \ldots, \xi_{d}\right) \rightarrow-G_{N} m / r_{3}$. Eq. (4) was widely used in the concept of large extra dimensions which gives possibility to solve the hierarchy problem [2,3]. It is also convenient to rewrite (4) via fundamental Planck scales:

$$
\frac{S_{D}}{S_{3}} \cdot M_{P l(4)}^{2}=M_{P l(\mathscr{D})}^{2+d} \prod_{i=1}^{d} a_{i},
$$

where $M_{P l(4)}=G_{N}^{-1 / 2}=1.2 \times 10^{19} \mathrm{GeV}$ and $M_{P l(\mathscr{D})} \equiv G_{\mathscr{D}}^{-1 /(2+d)}$ are fundamental Planck scales in four and $\mathscr{D}$ spacetime dimensions, respectively.

In opposite limit when all $a_{i} \rightarrow+\infty$ the sums in Eq. (3) can be replaced by integrals. Using the standard integrals (e.g. from [5]) and relation (4), we can easily show that, for example, in particular cases $d=1,2$ we get desire result: $\varphi_{D}\left(\mathbf{r}_{3}, \xi_{1}, \ldots, \xi_{d}\right) \rightarrow$ $-G_{\mathscr{D}} m /\left[(D-2) r_{3+d}^{1+d}\right]$.

In the case of one extra dimension $d=1$ we can perform summation of series in Eq. (3). To do it, we can apply the Abel-Plana formula or simply use the tables of series [5]. As a result, we arrive at compact and nice expression:

$$
\varphi_{4}\left(\mathbf{r}_{3}, \xi\right)=-\frac{G_{N} m}{r_{3}} \frac{\sinh \left(\frac{2 \pi r_{3}}{a}\right)}{\cosh \left(\frac{2 \pi r_{3}}{a}\right)-\cos \left(\frac{2 \pi \xi}{a}\right)},
$$

where $r_{3} \in[0,+\infty)$ and $\xi \in[0, a]$. It is not difficult to verify that this formula has correct asymptotes when $r_{3}>>a$ and $r_{4}<<a$. Fig. 1 demonstrates the shape of this potential. Dimensionless variables $\eta_{1} \equiv r_{3} / a \in[0,+\infty)$ and $\eta_{2} \equiv \xi / a \in[0,1]$. With respect to variable $\eta_{2}$, this potential has two minima at $\eta_{2}=0,1$ and one maximum at $\eta_{2}=1 / 2$. We continue the graph to negative values of $\eta_{2} \in[-1,1]$ to show in more detail the form of minimum at $\eta_{2}=0$. The potential (6) is finite for any value of $r_{3}$ if $\xi \neq 0, a$ and goes to $-\infty$ as $-1 / r_{4}^{2}$ if simultaneously $r_{3} \rightarrow 0$ and $\xi \rightarrow 0, a$ (see Fig. 2 ). We would like to mention that in particular case $\xi=0$ formula (6) was also found in [6].

Having at hand formulas (3) and (6), we can apply it for calculation of some elementary physical problems and compare obtained results with known Newtonian expressions. For a working approximation, it is usually sufficient to summarize in (3) up to the first Kaluza-Klein modes $\left|k_{i}\right|=1(i=1, \ldots, d)$. Then, the terms with the biggest periods $a_{i}$ give the main contributions. If all test bodies are on the same brane $\left(\xi_{i}=0\right)$ we obtain:

$$
\varphi_{D}\left(\mathbf{r}_{3}, \xi_{1}=0, \ldots, \xi_{d}=0\right) \equiv \varphi_{D}\left(r_{3}\right) \approx-\frac{G_{N} m}{r_{3}}\left[1+\alpha \exp \left(-\frac{r_{3}}{\lambda}\right)\right]
$$

where $\alpha=2 s(1 \leq s \leq d), \lambda=a /(2 \pi)$ and $s$ is a number of extra dimensions with periods of tori $a_{i}$ which are equal (or approximately equal) to $a=\max a_{i}$. If $a_{1}=a_{2}=$ $\ldots=a_{d}=a$, then $s=d$. Thus, the correction to Newton's potential has the form of 


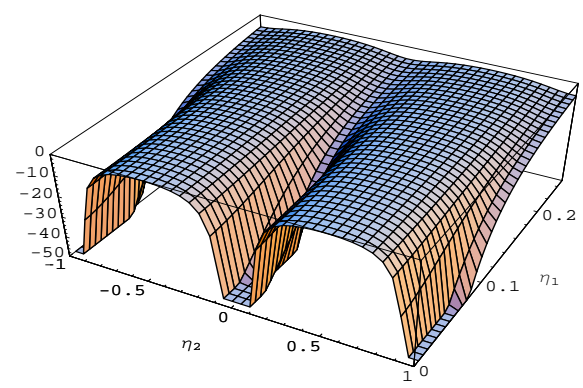

FIGURE 1. Graph of function $\tilde{\varphi}\left(\eta_{1}, \eta_{2}\right) \equiv \varphi_{4}\left(\mathbf{r}_{3}, \xi\right) /\left(G_{N} m / a\right)=-\sinh \left(2 \pi \eta_{1}\right) /\left[\eta_{1}\left(\cosh \left(2 \pi \eta_{1}\right)-\right.\right.$ $\left.\left.\cos \left(2 \pi \eta_{2}\right)\right)\right]$.

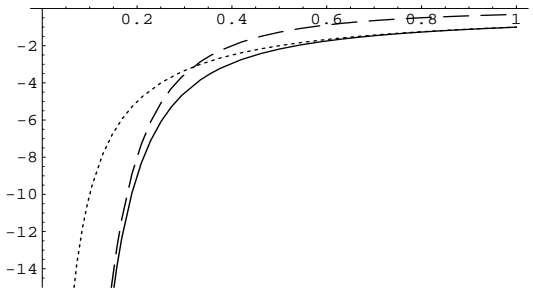

FIGURE 2. Section $\xi=0$ of potential (6). Solid line is $\tilde{\varphi}\left(\eta_{1}, 0\right)=-\sinh \left(2 \pi \eta_{1}\right) /\left[\eta_{1}\left(\cosh \left(2 \pi \eta_{1}\right)-1\right)\right]$ which goes to $-1 / \eta_{1}$ (dotted line) for $\eta_{1} \rightarrow+\infty$ and to $-1 /\left(\pi \eta_{1}^{2}\right)$ (dashed line) for $\eta_{1} \rightarrow 0$.

Yukawa potential. It is now customary to interpret tests of gravitational inverse-square law (ISL) as setting limits on additional Yukawa contribution. The overall diagram of the experimental constraints can be found in [1] (see Figure 5) and we shall use these data for limitation $a$ for given $\alpha$.

\section{APPLICATION $(d=1, \xi=0)$}

Now, we apply formula (6) to some particular geometrical configurations. For our calculations we shall use the case of $\xi=0$. It means that test bodies have the same coordinates in the extra dimension. It takes place e.g. when test bodies are on the same brane. Also, to get numerical results we should define the size $a$ of the extra dimension. If the standard model fields are not localized on the brane, then experiments give the upper bound $a \lesssim 10^{-17} \mathrm{~cm}^{1}$. This value $a$ can be greatly increased if we suppose that the standard model fields are localized on the brane. In this case, we can obtain the upper bound for $a$ from the gravitational inverse-square law experimental data depicted in Figure 5 of paper [1]. As we have shown above, $\alpha=2$ for $d=1$. For this value $\alpha$,

\footnotetext{
${ }^{1}$ Coulomb's law has been tested for separations down to $10^{-16} \mathrm{~cm}$ in $e^{+} e^{-}$leptonic interactions at highenergy colliders [1]. Nevertheless, see also the footnote 2 below.
} 
Figure 5 gives $\lambda \leq 2 \times 10^{-2} \mathrm{~cm} \Rightarrow a \leq 1.3 \times 10^{-1} \mathrm{~cm}$.

\section{Infinitesimally thin shell}

Let us consider first an infinitesimally thin shell of mass $m=4 \pi R^{2} \sigma$, where $R$ and $\sigma$ are radius and surface density of the shell. Then, gravitational potential of this shell in a point with radius vector $\mathbf{r}_{\mathbf{3}}$ (from the center of the shell) is

$$
\varphi_{4}\left(r_{3}\right)=-\frac{G_{N} \sigma R a}{r_{3}} \ln \left\{\frac{\cosh \left(\frac{2 \pi\left(r_{3}+R\right)}{a}\right)-1}{\cosh \left(\frac{2 \pi\left(r_{3}-R\right)}{a}\right)-1}\right\} .
$$

This formula demonstrates two features of the considered models. Firstly, we see that inside $\left(r_{3}<R\right)$ of the shell gravitational potential is not a constant. Thus, a test body undergoes an acceleration in contrast to the Newtonian case (and to Birkhoff's theorem of general relativity in four-dimensional spacetime which states that the metric inside an empty spherical cavity in the center of a spherically symmetric system is the Minkowski metric). Secondly, this potential has a logarithmic divergency when $r_{3} \rightarrow R$ : $\varphi_{4}\left(r_{3}\right) \approx-\frac{G_{N} m}{R}\left[1-\frac{a}{2 \pi R} \ln \left(2 \pi\left|R-r_{3}\right| / a\right)\right]$ where we took into account $R>>a$ and $\left|R-r_{3}\right|<<a$. For example, in the case $2 \pi R=10 \mathrm{~cm}$ and $2 \pi\left|R-r_{3}\right|=10^{-1} a$, the deviation constitutes $2.3 \times 10^{-2}$ and $2.3 \times 10^{-18}$ parts of the Newtonian value $-G_{N} m / R$ for $a=10^{-1} \mathrm{~cm}$ and $a=10^{-17} \mathrm{~cm}$, respectively. In principle, the former estimate is not very small. However, it is very difficult to set an experiment which satisfies the condition $\left|R-r_{3}\right|<<a$. If the shell has a finite thickness, then the divergence disappears.

\section{Spherical shell}

The gravitational potential of a spherical shell of inner radius $R_{1}$ and outer radius $R_{2}$ can be written as

$$
\varphi_{4}\left(r_{3}\right)=-\frac{G_{N} \rho a}{r_{3}} \int_{R_{1}}^{R_{2}} R \ln \left\{\frac{\cosh \left(\frac{2 \pi\left(r_{3}+R\right)}{a}\right)-1}{\cosh \left(\frac{2 \pi\left(r_{3}-R\right)}{a}\right)-1}\right\} d R,
$$

where $\rho$ is a constant volume density of the shell: $\rho=m /\left[\frac{4 \pi}{3}\left(R_{2}^{3}-R_{1}^{3}\right)\right]$. It is useful to present this potential in the form of series. For example, inside $\left(r_{3} \leq R_{1}\right)$ of the shell it reads:

$$
\begin{aligned}
\varphi_{4}\left(r_{3}\right) & =2 G_{N} \rho\left\{-\pi\left(R_{2}^{2}-R_{1}^{2}\right)+\frac{a^{2}}{\pi r_{3}} \sum_{k=1}^{+\infty} \frac{\sinh \left(\frac{2 \pi k}{a} r_{3}\right)}{k^{2}}\right. \\
& \left.\times\left[\left(R+\frac{a}{2 \pi k}\right) \exp \left(-\frac{2 \pi k}{a} R\right)\right]_{R_{1}}^{R_{2}}\right\}
\end{aligned}
$$


where we singled out zero mode $k=0$ which corresponds to the Newtonian limit. It can be easily seen that this series does not diverge when $r_{3} \rightarrow R_{1}$. However, acceleration of a test body diverges when it approaches boundaries $R_{1}$ and $R_{2}$ of the shell. It clearly follows e.g. from the form of the acceleration inside $\left(r_{3} \leq R_{1}\right)$ of the shell:

$$
\begin{aligned}
& -\frac{d \varphi_{4}}{d r_{3}}=\frac{2 G_{N} \rho a^{2}}{\pi r_{3}^{2}} \sum_{k=1}^{+\infty} \frac{1}{k^{2}}\left[-\frac{2 \pi k}{a} r_{3} \cosh \left(\frac{2 \pi k}{a} r_{3}\right)\right. \\
& \left.+\sinh \left(\frac{2 \pi k}{a} r_{3}\right)\right]\left[\left(R+\frac{a}{2 \pi k}\right) \exp \left(-\frac{2 \pi k}{a} R\right)\right]_{R_{1}}^{R_{2}} \geqslant 0
\end{aligned}
$$

and outside $\left(r_{3} \geq R_{2}\right)$ of the shell:

$$
-\frac{d \varphi_{4}}{d r_{3}}=-\frac{G_{N} m}{r_{3}^{2}}-\frac{G_{N} \rho a^{3}}{\pi^{2} r_{3}^{2}} \sum_{k=1}^{+\infty} \frac{\left(1+\frac{2 \pi k}{a} r_{3}\right)}{k^{3}} \exp \left(-\frac{2 \pi k}{a} r_{3}\right)\left[h_{k}\left(R_{2}\right)-h_{k}\left(R_{1}\right)\right]<0,
$$

where $h_{k}(R)=\frac{2 \pi k}{a} R \cosh \left(\frac{2 \pi k}{a} R\right)-\sinh \left(\frac{2 \pi k}{a} R\right)$. Divergence of acceleration originates from the divergence of the series $\sum_{k=1}^{+\infty} 1 / k$ and has the form $\pm 2 G_{N} \rho a \ln \frac{2 \pi \varepsilon}{a}$. Here, $\varepsilon=\left|R_{1,2}-r_{3}\right|$ and,-+ corresponds to $r_{3} \rightarrow R_{1}$ and $r_{3} \rightarrow R_{2}$, respectively. In the case of a sphere $\left(R_{1}=0\right.$ and $\left.R_{2} \equiv R\right)$ this divergence can be rewritten in the form $-3 \frac{G_{N} m}{R^{2}} \frac{a}{2 \pi R} \ln \left(2 \pi\left|R-r_{3}\right| / a\right)$. Similar to the case of the infinitesimally thin shell, this deviation from the Newtonian acceleration $-G_{N} m / R^{2}$ is also difficult to observe at experiments for considered above parameters. Eqs. (11) and (12) show that acceleration changes the sign from negative outside of the shell to positive inside of the shell (see Figure (3)). This change happens within the shell.

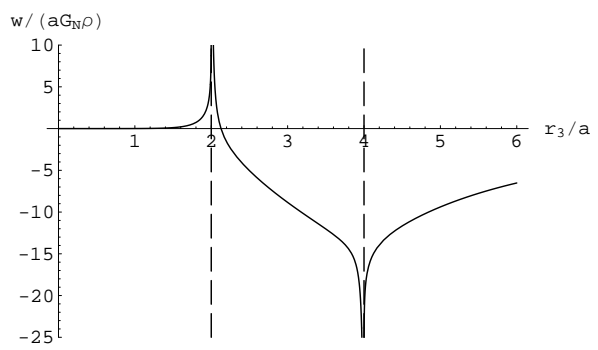

FIGURE 3. Graph of acceleration $w=-d \varphi_{4} / d r_{3}$ in dimensionless units (see Eqs. (11) and (12)). Here, $a=2.5, R_{1}=5$ and $R_{2}=10$. The dashed lines correspond to radii of the shell. The rightmost line goes to the Newtonian asymptote $-1 / r_{3}^{2}$ when $r_{3} \rightarrow+\infty$. 


\section{Gravitational self-energy of a sphere}

For a sphere of constant volume density $\rho$, mass $m$ and radius $R$, a gravitational selfenergy is

$$
\begin{aligned}
U & =2 \pi \rho \int_{0}^{R} r_{3}^{2} \varphi_{4}\left(r_{3}\right) d r_{3} \\
& =U_{N}\left\{1+15\left(\frac{a}{2 \pi R}\right)^{3} \sum_{k=1}^{+\infty} \frac{1}{k^{3}}\left[\frac{2 \pi k R}{3 a}+\left(\frac{a}{2 \pi k R}\right)^{2} F_{k}\right]\right\},
\end{aligned}
$$

where

$$
F_{k}=\left(1+\frac{2 \pi k R}{a}\right) \exp \left(-\frac{2 \pi k R}{a}\right)\left[\sinh \left(\frac{2 \pi k R}{a}\right)-\frac{2 \pi k R}{a} \cosh \left(\frac{2 \pi k R}{a}\right)\right]
$$

and $U_{N}=-3 G_{N} m^{2} /(5 R)$ is gravitational self-energy of a sphere in the Newtonian limit [7]. In the case $R>>a$, the difference $\triangle U \equiv U-U_{N}$ reads:

$$
\triangle U \approx U_{N} \frac{15}{8 \pi^{3}}\left(\frac{a}{R}\right)^{2} \frac{2 \pi}{3} \sum_{k=1}^{+\infty} \frac{1}{k^{2}}=U_{N} \frac{5}{24}\left(\frac{a}{R}\right)^{2},
$$

where we took into account $\sum_{k=1}^{\infty} 1 / k^{2}=\pi^{2} / 6$. Therefore, this difference is suppressed by power law (with respect to ratio between $a$ and $R$ ) but not exponentially as it is usually expected for Kaluza-Klein modes. Nevertheless, for Sun with $R \approx 7 \times 10^{10} \mathrm{~cm}$ this value is a negligible part of $U_{N}$ even if $a \approx 10^{-1} \mathrm{~cm}: \triangle U \approx 4 \times 10^{-25} U_{N}$ where $U_{N} \approx-2 \times 10^{48} \mathrm{erg}$ [7]. In the case of a neutron star with $R \approx 10^{6} \mathrm{~cm}$, the difference is $\triangle U \approx 2 \times 10^{-15} U_{N}$ for $a \approx 10^{-1} \mathrm{~cm}$.

\section{Gravitational interaction of two spheres}

Energy of gravitational interaction between two spheres of constant volume densities $\rho, \rho^{\prime}$, masses $m, m^{\prime}$ and radii $R, R^{\prime}$ on a distance $r_{3} \geq R+R^{\prime}$ reads

$$
U\left(r_{3}\right)=-\frac{G_{N} m m^{\prime}}{r_{3}}\left\{1+18\left(\frac{a}{2 \pi R}\right)^{3}\left(\frac{a}{2 \pi R^{\prime}}\right)^{3} \sum_{k=1}^{+\infty} \frac{1}{k^{6}} \exp \left(-\frac{2 \pi k}{a} r_{3}\right) h_{k}(R) h_{k}\left(R^{\prime}\right)\right\} .
$$

The member of the series with $k=1$ (first Kaluza-Klein mode) gives the main correction to the Newtonian expression and acquires the form of Yukawa potential. In this case, for the force of gravitational interaction between these two spheres we obtain:

$$
-\frac{d U}{d r_{3}} \approx-\frac{G_{N} m m^{\prime}}{r_{3}^{2}}\left\{1+\frac{9}{2}\left(\frac{a}{2 \pi R}\right)^{2}\left(\frac{a}{2 \pi R^{\prime}}\right)^{2} \frac{2 \pi r_{3}}{a} \exp \left[-\frac{2 \pi}{a}\left(r_{3}-R-R^{\prime}\right)\right]\right\} .
$$


where we made additional natural assumption $R, R^{\prime}>>a /(2 \pi)$. If $r-R-R^{\prime} \approx a$, then we get an estimate:

$$
-\frac{d U}{d r_{3}} \approx-\frac{G_{N} m m^{\prime}}{r_{3}^{2}}\left\{1+0.0084\left(\frac{a}{2 \pi R}\right)^{2}\left(\frac{a}{2 \pi R^{\prime}}\right)^{2} \frac{2 \pi r_{3}}{a}\right\} .
$$

For example, in the case $R=R^{\prime}=1 \mathrm{~cm}$ and $a /(2 \pi)=2 \times 10^{-2} \mathrm{~cm}$ the correction is $1.4 \times 10^{-7}$. In the case of the internal space topology $T^{d}$ the correction term in (18) acquires a pre-factor $s$ which satisfies the condition $1 \leq s \leq d$ and represents a number of extra dimensions with periods of the torus which are equal (or approximately equal) to $a=\max a_{i}$. If all of $a_{i}$ are equal to each other, $s=d$. Increasing the number of extra dimensions $d$, finally we arrive at the condition when the correction term becomes big enough to contradict with experimental data. Therefore, in this case we can get a limitation on a maximal number of extra dimensions for considered models. Certainly, the models with infinite number of extra dimensions with $a=\max a_{i}$ are forbidden.

It is worth of noting that all formulas obtained above can be also applied to the Coulomb's law if electromagnetic field is not localized on the brane.

\section{IY. SMEARED EXTRA DIMENSIONS}

In what follows, we consider asymmetrical extra dimension model (cf. [8]) with topology

$$
M_{D}=\mathbb{R}^{3} \times T^{d-p} \times T^{p}, \quad p \leq d,
$$

where we suppose that $(d-p)$ tori have the same "large" period $a$ and $p$ tori have "small" equal periods $b$. In this case, the fundamental Planck scale relation (5) reads

$$
\frac{S_{D}}{S_{3}} \cdot M_{P l(4)}^{2}=M_{P l(\mathscr{D})}^{2+d} a^{d-p} b^{p}
$$

Additionally, we assume that test bodies are uniformly smeared/spreaded over small extra dimensions. Thus, test bodies have a finite thickness in small extra dimensions (thick brane approximation). For short, we shall call such small extra dimensions as "smeared" extra dimensions. If $p=d$ then all extra dimensions are smeared.

It is not difficult to show that the gravitational potential does not feel smeared extra dimensions. We can prove this statement by three different methods. First, we can directly solve D-dimensional Poisson equation (1) with the periodic boundary conditions for the extra dimensions $\xi_{p+1}, \ldots, \xi_{d}$ and the mass density $\rho=\left(m / \prod_{i=1}^{p} a_{i}\right) \delta\left(\mathbf{r}_{3}\right) \boldsymbol{\delta}\left(\xi_{p+1}\right) \ldots \delta\left(\xi_{d}\right)$. Second, we can average solutions (3) and (6) over dimensions $\xi_{1}, \ldots, \xi_{p}$ and take into account that $\int_{0}^{a} \cos (2 \pi k \xi / a) d \xi=0$. In particular case of one extra dimensional, we can also show that

$$
-\frac{G_{N} m}{a r_{3}} \sinh \left(\frac{2 \pi r_{3}}{a}\right) \int_{0}^{a}\left[\cosh \left(\frac{2 \pi r_{3}}{a}\right)-\cos \left(\frac{2 \pi \xi}{a}\right)\right]^{-1} d \xi=-\frac{G_{N} m}{r_{3}}
$$


Finally, it is clear that in the case of test masses smeared over extra dimensions, the gravitational field vector $\mathbf{E}_{D}=-\nabla_{D} \varphi_{D}$ does not have components with respect to extra dimensions: $\mathbf{E}_{D}=E_{D} \mathbf{n}_{r_{3}}$. Thus, applying the Gauss's flux theorem to the Poisson equation, we obtain: $E_{D}\left(r_{3}\right)=-G_{N} m / r_{3}^{2} \rightarrow \varphi_{D}\left(r_{3}\right)=-G_{N} m / r_{3}$. Therefore, all these 3 approaches show that in the case of $p$ smeared extra dimensions the wave numbers $k_{1}, \ldots, k_{p}$ disappear from Eq. (3) and we should perform summation only with respect to $k_{p+1}, \ldots, k_{d}$.

\section{Effective gravitational constant}

As it follows from Eq. (17), in the Yukawa approximation, the gravitational force between two spheres with masses $m_{1}, m_{2}$, radii $R_{1}, R_{2}$ and distance $r_{3}$ between the centers of the spheres reads:

$$
F=-\frac{G_{N(e f f)} m_{1} m_{2}}{r_{3}^{2}}
$$

where

$$
\begin{aligned}
G_{N(e f f)}\left(r_{3}\right) & \approx G_{N}\left\{1+\frac{9}{2} s\left(\frac{a}{2 \pi R_{1}}\right)^{2}\left(\frac{a}{2 \pi R_{2}}\right)^{2} \frac{2 \pi r_{3}}{a} \exp \left[-\frac{2 \pi}{a}\left(r_{3}-R_{1}-R_{2}\right)\right]\right\} \\
& \equiv G_{N}\left(1+\delta_{G}\right)
\end{aligned}
$$

is an effective gravitational constant. Here, the pre-factor $s$ appeared due to generalization of formula (17) to the case of the internal space topology $T^{d}$. If we consider a model with $p$ smeared extra dimensions, $s$ in Eq. (23) is replaced by $(d-p)$. Now, we want to evaluate the corrections $\delta_{G}$ to the Newton's gravitational constant and to estimate their possible influence on the experimental data. As it follows from Figure 2 in the CODATA 2006, the most precise values of $G_{N}$ were obtained in the University Washington and the University Zürich experiments $[9,10]$. They are $G_{N} / 10^{-11} \mathrm{~m}^{3} \mathrm{~kg}^{-1} \mathrm{~s}^{-2}=$ $6.674215 \pm 0.000092$, and $6.674252 \pm 0.000124$, respectively. Let us consider two particular examples: $(\mathscr{D}=5)$-dimensional model with $d=1, p=0 \rightarrow \alpha=2$ and $(\mathscr{D}=10)$ dimensional model with $d=6, p=3 \rightarrow \alpha=6$. For these values of $\alpha$, Figure 5 in [1] gives the upper limits for $\lambda=a /(2 \pi)$ correspondingly $\lambda \approx 2 \times 10^{-2} \mathrm{~cm}$ and $\lambda \approx 1.3 \times 10^{-2} \mathrm{~cm}$. To calculate $\delta_{G}$, we take parameters of the Moscow experiment [11]: $R_{1} \approx 0.087 \mathrm{~cm}$ for a platinum ball with the mass $m_{1}=59.25 \times 10^{-3} \mathrm{~g}, R_{2} \approx 0.206 \mathrm{~cm}$ for a tungsten ball with the mass $m_{2}=706 \times 10^{-3} \mathrm{~g}$ and $r_{3}=0.3773 \mathrm{~cm}$. For both of these models we obtain $\delta_{G} \approx 0.0006247$ and $\delta_{G} \approx 0.0000532$, respectively. Both of these values are very close to the measurement accuracy of $G_{N}$ in $[9,10]$. So, if the same accuracy can be achieved in the Moscow-type experiments, then, changing values of $R_{1,2}$ and $r_{3}$, we can reveal extra dimensions or obtain experimental limitations on considered models. 


$$
\text { Model: } \mathscr{D}=10 \text { with } d=6, p=3
$$

Let us consider in more detail $(\mathscr{D}=10)$-dimensional model with 3 smeared dimensions. Here, we have very symmetrical with respect to a number of spacial dimensions structure: 3 our external dimensions, 3 large extra dimensions with periods $a$ and three small smeared extra dimensions with periods $b$. For $b$ we put a limitation: $b \leq b_{\max }=10^{-17} \mathrm{~cm}$ which is usually taken for thick brane approximation ${ }^{2}$. As we mentioned above, in the case of $\alpha=6$, for $a$ we should take a limitation $a \leq a_{\max }=8.2 \times 10^{-2} \mathrm{~cm}$. To solve the hierarchy problem, the multidimensional Planck scale is usually considered from $1 \mathrm{TeV}$ up to approximately $130 \mathrm{TeV}$ (see e.g. [8, 12]). To make some estimates, we take $M_{\min }=1 \mathrm{TeV} \lesssim M_{P l(10)} \lesssim M_{\max }=50 \mathrm{TeV}$. Thus, as it follows from Eq. (20), the allowed values of $a$ and $b$ should satisfy inequalities:

$$
\frac{S_{9}}{S_{3}} \frac{M_{P l(4)}^{2}}{M_{\text {max }}^{8}} \leq a^{3} b^{3} \leq \frac{S_{9}}{S_{3}} \frac{M_{P l(4)}^{2}}{M_{\text {min }}^{8}}
$$

Counting all limitations, we find allowed region for $a$ and $b$ (shadow area in Fig. (4)). In this trapezium, the upper horizontal and right vertical lines are the decimal logarithms of $a_{\max }$ and $b_{\max }$, respectively. The right and left inclined lines correspond to $M_{P l(10)}=1 \mathrm{TeV}$ and $M_{P l(10)}=50 \mathrm{TeV}$, respectively. To illustrate this picture, we consider two points $\mathrm{A}$ and $\mathrm{B}$ on the line $M_{P l(10)}=1 \mathrm{TeV}$. Here, we have $a=0.82 \times 10^{-1} \mathrm{~cm}$, $b=10^{-21.5} \mathrm{~cm}$ for A and $a=10^{-4} \mathrm{~cm}, b=10^{-18.6} \mathrm{~cm}$ for $\mathrm{B}$. These values of large extra dimensions $a$ are much bigger than in the standard approach $a \sim 10^{(32 / 6)-17} \mathrm{~cm}$ $\approx 10^{-11.7} \mathrm{~cm}[2,3]$.

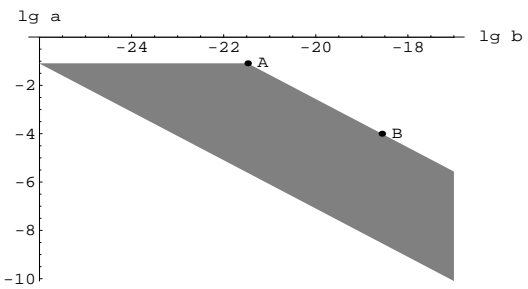

FIGURE 4. Allowed region (shadow area) for periods of large $(a)$ and smeared $(b)$ dimensions in the model $\mathscr{D}=10$ with $d=6, p=3$.

\section{Model: $\mathscr{D}$-arbitrary and $d=p$}

In this model, the test masses are smeared over all extra dimensions. Therefore, in non-relativistic limit, there is no deviation from the Newton's law at all. It worth of

\footnotetext{
2 This limitation follows from the electrical inverse-square law experiments. However, in the case of the smeared extra dimensions, the ISL is not sensitive to these dimensions. Thus, it gives us a possibility to relax considerably this bound.
} 
noting, that this result does not depend on the size of smeared extra dimensions. The ISL experiments will not show any deviation from the Newton's law without regard to the size $b$ (see also Eq. (23) where $s=d-p=0$ ). Similar reasoning are also applicable to Coulomb's law. It is necessary to suggest other experiments which can reveal the multidimensionality of our spacetime. Nevertheless, we can solve the hierarchy problem in this model because Eq. (20) (where $d=p$ ) still works. For example, in the case of bosonic string dimension $\mathscr{D}=26$ we find $M_{P l(26)} \approx 31 \mathrm{TeV}$ for $b=10^{-17} \mathrm{~cm}$. In the case $\mathscr{D}=10$ we get $M_{P l(10)} \approx 30 \mathrm{TeV}$ for $b=5.59 \times 10^{-14} \mathrm{~cm}$.

\section{Y. CONCLUSIONS}

We have considered generalization of the Newton's potential to the case of extra dimensions where multidimensional space has topology $M_{D}=\mathbb{R}^{3} \times T^{d}$. We obtained the exact solution which describes smooth transition from the Newtonian behavior $1 / r_{3}$ for distances bigger than periods of tori (the extra dimension sizes) to multidimensional be-

havior $1 / r_{D}^{D-2}$ in opposite limit. In the case of one extra dimension, the gravitational potential is expressed via compact and elegant formula (6). Then, this formula was applied to an infinitesimally thin shell, a shell, a sphere and two spheres to get gravitational potentials and acceleration of a test body for these configurations and to compare obtained results with the known Newtonian formulas. In some cases, obtained potentials and accelerations have logarithmic divergences near the boundaries of shells and spheres. Additionally, in contrast to the Newtonian case, test bodies accelerate inside of shells. For each considered problems, we found deviations from the known Newtonian expressions.

As an Yukawa potential approximation, it was shown that the corrections to the gravitational constant in the Cavendish-type experiment can be within the measurement accuracy of $G_{N}$. It may reveal the extra dimensions or provide experimental limitations on parameters of multidimensional models.

Then, we proposed models where test masses can be smeared over extra dimensions. In this case, the gravitational potential does not feel smeared dimensions. The number of smeared dimensions can be equal or less than the total number of the extra dimensions. Such approach opens new remarkable possibilities.

For example in the case $\mathscr{D}=10$ with 3 large and 3 smeared extra dimensions and $M_{P l(10)}=1 \mathrm{TeV}$, the large extra dimensions can be as big as the upper limit established by the ISL experiments for $\alpha=6$, i.e. $a \approx 0.82 \times 10^{-1} \mathrm{~cm}$. This value of $a$ is in many orders of magnitude bigger than the rough estimate $a \approx 10^{-11.7} \mathrm{~cm}$ obtained from the fundamental Planck scale relation of the form of Eq. (5).

The limiting case where all extra dimensions are smeared is another interesting example. Here, there is no deviation from the Newton's law at all. Nevertheless, the hierarchy problem can be solved in this model. 


\section{ACKNOWLEDGMENTS}

A. Zh. acknowledges the hospitality of the Theory Division of CERN and the High Energy, Cosmology and Astroparticle Physics Section of the ICTP during preparation of this work. This work was supported in part by the "Cosmomicrophysics" programme of the Physics and Astronomy Division of the National Academy of Sciences of Ukraine.

\section{REFERENCES}

1. E.G. Adelberger, B.R. Heckel and A.E. Nelson, Ann. Rev. Nucl. Part. Sci. 53, 77 (2003); arXiv:hep$\mathrm{ph} / 0307284$.

2. N. Arkani-Hamed, S. Dimopoulos and G. Dvali, Phys. Lett. B 429, 263 (1998); hep-ph/9803315. I. Antoniadis, N. Arkani-Hamed, S. Dimopoulos and G. Dvali, Phys. Lett. B 436, 257 (1998); arXiv:hep$\mathrm{ph} / 9804398$.

3. N. Arkani-Hamed, S. Dimopoulos and G. Dvali, Phys. Rev. D 59, 086004 (1999); arXiv:hep$\mathrm{ph} / 9807344$.

4. P. Callin and C.P. Burgess, Nucl. Phys. B 752, 60 (2006); arXiv:hep-ph/0511216.

5. A.P. Prudnikov, Yu.A. Brychkov and O.I. Marichev, Integrals and Series, vol. 1: Elementary Functions, (Gordon and Breach Science Publishers, New York, 1986).

6. A. O. Barvinsky and S. N. Solodukhin, Nucl. Phys. B 675, 159 (2003); arXiv:hep-th/0307011 .

7. Ch.Kittel, W. D. Knight and M. A. Ruderman, Mechanics: Berkeley Physics Course. vol. 1, (McGrawHill, New York, 1965).

8. J. Lykken and S. Nandi, Phys.Lett. B 485, 224 (2000); arXiv:hep-ph/9908505.

9. J.H. Gundlach and S.M. Merkowitz, Phys.Rev.Lett. 85, 2869 (2000); arXiv:gr-qc/0006043.

10. St. Schlamminger et al., Phys.Rev. D 74, 082001 (2006); arXiv:hep-ex/0609027.

11. V.P. Mitrofanov and O.I. Ponomareva, Sov. Phys. JETP bf 67, 1963 (1988).

12. S. Hannestad and G. Raffelt, Phys. Rev. Lett. 87, 051301 (2001); arXiv:hep-ph/0103201. 